\title{
ESTIMULACIÓN DE NARRACIONES INFANTILES
}

\section{Children narrative intervention}

\author{
Mariangela Maggiolo Landaeta ${ }^{(1)}$, Carmen Julia Coloma Tirapegui (2), \\ María Mercedes Pavez Guzmán ${ }^{(3)}$
}

\begin{abstract}
RESUMEN
Objetivo: proveer evidencia sobre la contribución de estimular los guiones para el desarrollo narrativo en un grupo de niños con Trastorno Específico de Lenguaje y déficit narrativo. Métodos: seis niños con Trastorno Específico del Lenguaje constituyeron el grupo experimental al que se le aplicó el programa y seis niños con Trastorno Específico del lenguaje conformaron el grupo control. Todos presentaban déficit narrativo y tenían un promedio de edad de 4 años, 5 meses. El programa se aplicó individualmente durante 18 sesiones. Se comparó el desempeño narrativo antes y después de la intervención en ambos grupos de niños. Resultados: todos los niños del grupo experimental incrementaron significativamente su desarrollo narrativo. Incluso, el $50 \%$ de ellos logró niveles narrativos correspondientes a su edad cronológica. En el grupo control no se observó un aumento significativo. Conclusiones: el programa basado en los guiones es una estrategia eficaz para estimular las habilidades narrativas tempranas en niños con Trastorno Específico del Lenguaje y déficit narrativo.
\end{abstract}

DESCRITORES: Trastornos del Lenguaje; Narración; Terapia del Lenguaje

\section{INTRODUCCIÓN}

El Trastorno Específico del Lenguaje (TEL) es un cuadro que por sus características y frecuencia ha despertado gran interés en la investigación de las dificultades infantiles del lenguaje. Se estima que entre el $2 \%$ y el $7 \%$ de la población lo presenta ${ }^{1,2}$.

Se define como un déficit en el desarrollo del lenguaje en ausencia de factores que puedan explicarlo, por lo tanto, los niños no deben evidenciar problemas neurológicos, auditivos o sociofamiliares ${ }^{3,4}$. Lo anterior implica realizar un diagnóstico por exclusión, es decir, establecer la ausencia de este tipo de alteraciones en el proceso de identificación del cuadro.

Los niños con TEL presentan una sintomatología lingüística muy diversa que puede involucrar

(1) Fonoaudióloga; Profesora Asociada de la Escuela de Fonoaudiología, Facultad de Medicina da la Universidad de Chile, Santiago, Chile.

(2) Linguista, Magíster en Educación Especial por la Pontificia Universidad Católica de Chile; Profesora Asociada de la Escuela de Fonoaudiología, Facultad de Medicina de la Universidad de Chile, Santiago, Chile.

(3) Lingüista; Profesora Asociada de la Escuela de Fonoaudiología, Facultad de Medicina de la Universidad de Chile, Santiago, Chile.

Conflito de interesse: inexistente desde dificultades principalmente expresivas hasta un serio compromiso en la comprensión y expresión verbal $^{5}$. Los síntomas más frecuentes son las dificultades fonológicas, el déficit morfosintáctico y los problemas de adquisición del léxico. En los últimos años se han incorporado además evidencia acerca de los déficits que presentan en determinadas habilidades cognitivas ${ }^{6,7}$.

Otro aspecto que se ha considerado en el estudio del TEL es el discurso narrativo, dada su relevancia como organizador de la experiencia humana ${ }^{8}$ y por su influencia en el aprendizaje de la lectura, especialmente en la comprensión lectora ${ }^{9}$. Esto último ha sido corroborado en preescolares con TEL en los que se ha determinado que sus relatos son un buen predictor para el éxito escolar ${ }^{10}$.

En este sentido, se constató que estos niños desarrollan más tardíamente sus narraciones que los con desarrollo típico del lenguaje ${ }^{11-13}$. También presentan dificultades para ordenar una historia, reconocer al protagonista y respetar la secuencia de los eventos ${ }^{10}$.

Otras investigaciones muestran que en la etapa escolar niños con antecedentes de problemas de lenguaje, quienes aparentemente han superado sus dificultades lingüísticas, presentan déficit en sus habilidades narrativas ${ }^{14}$. También manifiestan problemas en la coherencia y en la superestructura 
de sus narraciones ${ }^{15} \mathrm{y}$ en una proporción importante de ellos se observa un desempeño narrativo descendido ${ }^{16}$.

Estudios sobre desarrollo narrativo en niños sin problemas de lenguaje han permitido determinar una secuencia evolutiva en la que se distinguen tres etapas: no-estructuración, transición y estructuración de relatos. Las definiciones operacionales de la estructura narrativa se encuentran en la Figura 1.

La etapa de no-estructuración implica que en los relatos que efectúan, los niños no utilizan las categorías formales. Este período es característico de los pequeños de tres años. En la etapa de transición se producen secuencias de eventos que insinúan la aparición de categorías formales. Este estadio no es característico de una edad determinada, aunque se observa en algunos niños de cuatro y cinco años. Finalmente, la etapa de estructuración se caracteriza porque las narraciones infantiles se organizan sobre la base de las tres categorías formales básicas (presentación, episodio y final). Se presenta alrededor de los cuatro años y continúa desarrollándose en edades posteriores. Para el detalle sobre las etapas propuestas, ver Figura 2.

En relación a los estadios iniciales del desarrollo narrativo se ha observado que la experiencia con los scripts o guiones a edades tempranas favorece la adquisición de la estructura narrativa ${ }^{17}$. Por lo tanto, es importante un adecuado manejo de este tipo de rutinas en los niños pequeños ${ }^{18}$.
Los guiones son patrones lingüísticos y no lingüísticos que refieren a una experiencia habitual en la vida infantil y corresponden a una secuencia predecible de hechos que se suceden en el tiempo. Implican un conocimiento estereotipado que reduce la demanda cognitiva requerida para interactuar en una situación comunicativa ${ }^{19}$.

En el período preescolar, los niños participan de este tipo de eventos regulares y sistemáticos que les posibilitan formar una representación mental de esos sucesos. Por ello, desde pequeños pueden enunciarlos lingüísticamente ${ }^{20}$.

Entre los dos y tres años los niños transitan desde un juego basado en la organización narrativa que representa situaciones que ocurren en la vida real, a los relatos ficticios que se sustentan completamente en el lenguaje ${ }^{8}$.

Existen diversas estrategias terapéuticas que consideran el guión como un contenido importante en la estimulación del lenguaje y de la narración en particular. Al respecto, se describe la terapia del script ${ }^{21}$ como uno de los procedimientos de orientación naturalista en la intervención del lenguaje. De este modo, las rutinas familiares son el contexto fundamental para la estimulación lingüística, pues recrean situaciones reales en las que el niño participa habitualmente.

Por otra parte, se menciona el uso de los guiones asociados a situaciones de juegos como una técnica específica para facilitar el desarrollo de la narración, particularmente usada en preescolares ${ }^{19}$.

\section{Presentación}

a) Completa: personaje + problema.

b) Incompleta: solo personaje o solo problema.

a) Ejemplo: Era una ardillita (personaje) + le robaba la comida a sus amiguitos (problema). b) Ejemplo: Era una ardillita glotona (solo personaje).

Se comía la comida de sus amiguitos (solo problema).

\section{Episodio}

a) Completo: acción + obstáculo + resultado.

b) Incompleto: acción + obstáculo.

c) Incompleto: obstáculo + resultado a) Ejemplo: Los amiguitos le hicieron una casa chiquitita con muchos dulces y ella entró a la casa y se comió todo y se puso gorda (acciones y un estado) + no pudo salir de la casa (obstáculo) + se puso triste (resultado).

b) Ejemplo:

Se comió todo y se puso gorga (acción y estado) + no pudo salir (obstáculo)

c) No pudo salir ( obstáculo) + se puso triste (resultado).

\section{Final}

Se resuelve el problema de la presentación.
Ejemplo: Prometió no robarse más la comida de sus amiguitos.

Figura 1 - Definiciones operacionales de las categorías formales del cuento 
También, se ha desarrollado un plan ${ }^{22}$ que incluye una serie de actividades destinadas al manejo de esta clase de sucesos. Así, se enseña a los menores habilidades narrativas iniciales a través de dramatizaciones, ordenación de láminas y verbalizaciones.

En síntesis, la experiencia con los guiones contribuye al desarrollo narrativo temprano. Por ello, es factible plantear propuestas de intervención para preescolares con TEL que contemplen este contenido con el fin de favorecer el desarrollo narrativo y contribuir a un mejor desempeño académico en su futura vida escolar.

Según lo anterior, el propósito de este trabajo es presentar una experiencia de estimulación del manejo de guiones y su impacto en el desarrollo narrativo de niños con TEL.

\section{MÉTODOS}

El presente estudio es un proyecto financiado por la Escuela de Fonoaudiología de la Universidad de Chile y se realizó durante el año 2006. El diseño de investigación corresponde a un trabajo longitudinal cuasi experimental en el que se estudian los efectos de un tipo de intervención basada en los scripts, aplicada por un período de tiempo, sobre el desarrollo narrativo de niños con TEL que asisten a escuelas de lenguaje.

Para la selección de los sujetos se utilizaron los criterios de exclusión que se consideran habitualmente en el diagnóstico de $\mathrm{TEL}^{3}$. Con tal fin se revisaron las fichas escolares de los niños constatando su normalidad auditiva, neurológica y orofacial. Además, se corroboró que los participantes presentaran habilidades cognitivas dentro de rangos típicos. Desde la perspectiva verbal, se incluyeron solo aquellos niños con dificultades fonológicas, morfosintácticas, déficit en el desarrollo narrativo y comprensión dentro de rangos normales.

La evaluación cognitiva se realizó a todos los niños con la Escala de Madurez Mental de Columbia. Según el marco conceptual de la prueba se entiende esta capacidad como la resolución de tareas de clasificación perceptiva simple y la manipulación abstracta de conceptos simbólicos ${ }^{23}$. Se considera apropiada para niños con dificultades de lenguaje, pues no requiere de respuestas verbales.

Las características del desempeño verbal de los participantes se evaluaron con pruebas para la expresión en el plano fonológico, morfosintáctico y narrativo así como para la comprensión del lenguaje oral.

El componente fonológico se evaluó con el Test de Evaluación de los Procesos de Simplificación Fonológica (TEPROSIF) ${ }^{24}$. Este instrumento da cuenta de la cantidad de simplificaciones fonológicas que realizan los niños. El TEPROSIF es un teste que permite cuantificar el número de simplificaciones en base a la producción de 36 palabras estímulos. Por lo tanto, a mayor puntaje obtenido más cantidad de procesos fonológicos de simplificación, lo que evidencia la presencia de dificultades fonológicas. Todos los participantes rindieron sobre una desviación estándar en referencia a la media según su rango de edad $(X=17.5$; D.S. $=3.8)$ constándose así el problema fonológico.

Por otra parte, las dificultades gramaticales se evaluaron con la subprueba expresiva del Teste Exploratorio de Gramática Española de A. Toronto ${ }^{25}$. El desempeño gramatical de los niños se ubicó en el percentil 25, en el percentil 10 y bajo el percentil 10 según su rango de edad. Los niños con rendimiento inferior al percentil 10 se consideraron con un déficit gramatical.

La comprensión verbal se determinó con el Teste de Comprensión Auditiva de E. Carrow según una aplicación experimental efectuada en Chile ${ }^{26}$. El rendimiento de los niños se distribuyó entre el percentil 25 y 90 de acuerdo a la edad. Por lo tanto, presentaban un adecuado desempeño en comprensión.

El déficit narrativo se estableció ubicando a los niños en las etapas del desarrollo correspondiente según la Escala de Desarrollo Narrativo, ver Figura 2. Se seleccionaron aquellos menores que se encontraban en los primeros estadios del desarrollo narrativo (narraciones sin estructura). Los relatos se elicitaron a partir del recontado de historias sin apoyo de láminas ${ }^{27}$. Se utilizaron tres cuentos con las categorías formales de presentación, episodio $y$ final ${ }^{28}$.

Después de efectuado el proceso de evaluación se seleccionaron 12 niños con TEL expresivo y déficit en el discurso narrativo que se distribuyeron aleatoriamente en dos grupos: uno experimental y uno control.

Con el propósito de establecer si ambos grupos presentaban un desempeño narrativo similar antes de iniciar la intervención se comparó su rendimiento con la prueba no paramétrica de Mann-Whitney. No se evidenciaron diferencias significativas entre los grupos ( $U$ de Mann Whitney $=14.00$ ). Para ello, fue necesario analizar las narraciones infantiles y asignar puntaje a cada desempeño.

El análisis se basa en criterios estructurales. Así, la primera distinción es establecer si los cuentos están o no estructurados. Los relatos no estructurados son los que no presentan ninguna de las categorías básicas del discurso narrativo. Por el contrario, las narraciones infantiles estructuradas son las que evidencian al menos presentación y episodio. 


\begin{tabular}{|c|c|c|c|c|}
\hline & $\begin{array}{c}\text { NIVEL DE } \\
\text { DESEMPEÑO } \\
\text { NARRATIVO }\end{array}$ & CARACTERÍSTICAS & EDAD \\
\hline \multirow{4}{*}{\multicolumn{2}{|c|}{ NO ESTRUCTURA }} & 1 & NO DICE NADA & \multirow{3}{*}{3 AÑOS } \\
\hline & & 2 & DICE SOLO UNA ORACIÓN & \\
\hline & & 3 & $\begin{array}{c}\text { AGLUTINA SECUENCIAS DE ACCIONES } \\
\text { Y/O ESTADOS }\end{array}$ & \\
\hline & & 4 & $\begin{array}{l}\text { AGRUPA ENUMERATIVAMENTE } \\
\text { EN TORNO A UN PERSONAJE }\end{array}$ & \\
\hline \multirow{2}{*}{\multicolumn{2}{|c|}{ TRANSICIÓN }} & 5 & $\begin{array}{c}\text { HACE SECUENCIAS: } \\
\text { ACCIÓN + OBSTÁCULO Y/O } \\
\text { OBSTÁCULO + RESULTADO }\end{array}$ & \\
\hline & & 6 & $\begin{array}{l}\text { PRESENTACIÓN Y EPISODIO: } \\
\text { AMBOS INCOMPLETOS }\end{array}$ & \\
\hline \multirow{7}{*}{$\begin{array}{c}E \\
S \\
T \\
R \\
U \\
C \\
T \\
U \\
R \\
A\end{array}$} & \multirow{2}{*}{$\begin{array}{l}\text { I. RELATA CON } \\
\text { PRESENTACION } \\
\text { Y EPISODIO, } \\
\text { SOLO UNO DE } \\
\text { ELLOS } \\
\text { COMPLETO }\end{array}$} & 7 & $\begin{array}{l}\text { PRESENTACIÓN COMPLETA } \\
\text { Y EPISODIO INCOMPLETO } \\
\end{array}$ & \multirow{3}{*}{4 AÑOS } \\
\hline & & 8 & $\begin{array}{l}\text { PRESENTACIÓN INCOMPLETA } \\
\text { Y EPISODIO COMPLETO }\end{array}$ & \\
\hline & \multirow{2}{*}{$\begin{array}{l}\text { II. RELATA CON } \\
\text { PRESENTACIÓN } \\
\text { COMPLETA+ } \\
\text { EPISODIO } \\
\text { COMPLETO, } \\
\text { PERO SIN FINAL }\end{array}$} & 9 & $\begin{array}{c}\text { PRESENTACIÓN Y EPISODIO: } \\
\text { AMBOS COMPLETOS } \\
\text { (SIN FINAL) }\end{array}$ & \\
\hline & & 10 & $\begin{array}{l}\text { PRESENTACIÓN COMPLETA } \\
\text { CON ATRIBUTO Y EPISODIO } \\
\text { COMPLETO (SIN FINAL) }\end{array}$ & \multirow{3}{*}{5 Y 6 AÑOS } \\
\hline & \multirow{3}{*}{$\begin{array}{l}\text { III. RELATA CON } \\
\text { PRESENTACION } \\
\text { COMPLETA, } \\
\text { EPISODIO } \\
\text { COMPLETO Y } \\
\text { FINAL }\end{array}$} & 11 & $\begin{array}{c}\text { PRESENTACIÓN Y EPISODIO: } \\
\text { AMBOS COMPLETOS } \\
\text { MÁS FINAL }\end{array}$ & \\
\hline & & 12 & $\begin{array}{c}\text { PRESENTACIÓN COMPLETA } \\
\text { CON ATRIBUTO } \\
\text { ESPISODIO COMPLETO Y FINAL }\end{array}$ & \\
\hline & & 13 & $\begin{array}{c}\text { PRESENTACIÓN COMPLETA } \\
\text { CON ATRIBUTO } \\
\text { ESPISODIO COMPLETO CON META Y FINAL }\end{array}$ & 10 AÑOS \\
\hline
\end{tabular}

Figura 2 - Escala de desarrollo narrativo (desempeño evaluado con recontado de 3 cuentos)

Cuando los relatos infantiles carecen de estructura, se consignan los siguientes niveles con sus respectivos puntajes: no dice nada (0 puntos), dice una o dos oraciones ( 0.25 puntos), aglutina acciones sin relación ( 0.5 puntos), agrupa acciones en torno a un personaje ( 0.75 puntos) o produce secuencias de acción + obstáculo / obstáculo + resultado $(0.75$ puntos).

En las narraciones estructuradas se distinguen las categorías básicas del cuento infantil (presentación, episodio y final). A su vez, se identifica presentaciones y episodios completos e incompletos y a cada uno se le asignan puntajes. La presentación completa está constituida por personaje y problema (1 punto) y la incompleta, por uno de los dos elementos ( 0.5 puntos). Por su parte, los episo- dios completos están conformados por la secuencia acción + obstáculo + resultado (2 puntos) y los incompletos, por la secuencia acción + obstáculo / obstáculo + resultado (1 punto). Además, a la presencia del elemento meta tiene un puntaje adicional (2 puntos). Para la descripción de todos los niveles ver Figura 2.

Cada grupo estuvo constituido por 6 menores, tres niñas y tres niños en el grupo experimental con un promedio de edad de 4.5 años y un rango entre 4.1 y 4.7 años. El grupo control lo conformaron cinco niños y una niña, con un promedio de edad de 4.5 años y un rango entre 4.0 y 4.9 años.

Los rendimientos obtenidos por los participantes en cada una de las pruebas aplicadas se sintetizan en la Figura 3. 


\begin{tabular}{|c|c|c|c|c|c|}
\hline $\begin{array}{c}\text { Grupo } \\
\text { Experimental }\end{array}$ & $\begin{array}{c}\text { Escala } \\
\text { Columbia }\end{array}$ & TECAL & $\begin{array}{c}\text { Test } \\
\text { Exploratorio } \\
\text { de Gramática } \\
\text { Española de } \\
\text { A. Toronto }\end{array}$ & TEPROSIF & $\begin{array}{c}\text { Evaluación } \\
\text { del desarrollo } \\
\text { narrativo }\end{array}$ \\
\hline 1 & 32 & $\mathrm{p} 75$ & $\mathrm{p} 10$ & 29 & 1.5 \\
\hline 2 & 36 & $\mathrm{P} 50$ & $\mathrm{p} 10$ & 36 & 2.25 \\
\hline 3 & 34 & $\mathrm{P} 50-75$ & $\mathrm{p} 10$ & 30 & 0 \\
\hline 4 & 36 & $\mathrm{P} 50-75$ & $\mathrm{p} \mathrm{25}$ & 45 & 0 \\
\hline 5 & 36 & $\mathrm{P} 25-50$ & $\mathrm{p} \mathrm{25}$ & 40 & 0 \\
\hline 6 & 43 & $\mathrm{P} 75-90$ & $\mathrm{p} 10$ & 30 & 0 \\
\hline Grupo Control & & & & & 0 \\
\hline 1 & 34 & $\mathrm{P} 75-90$ & $\mathrm{p} 15$ & 56 & 0.75 \\
\hline 2 & 42 & $\mathrm{P} 75-90$ & $\mathrm{p} 10$ & 42 & 0.25 \\
\hline 3 & 43 & $\mathrm{P} 50-75$ & $\mathrm{p} 10$ & 31 & 0.5 \\
\hline 4 & 38 & $\mathrm{P} 25-50$ & $>\mathrm{p} 10$ & 30 & 0.5 \\
\hline 5 & 38 & $\mathrm{P} 25$ & $>\mathrm{p} 10$ & 30 & 0.5 \\
\hline 6 & 35 & $\mathrm{P} 75-90$ & $>\mathrm{p} 10$ & 28 & \\
\hline
\end{tabular}

Figura 3 - Rendimiento de los sujetos en las pruebas aplicadas al inicio del programa

Los procedimientos de este estudio se organizan fundamentalmente en función de las características del programa y su aplicación.

\section{Características del programa}

El programa de estimulación está compuesto por dos unidades que incluyen un contenido específico, objetivos y sugerencias de actividades.

El contenido central de la primera unidad corresponde a secuencias de eventos que forman parte de un guión, por lo que no se incluyen scripts completos. Su objetivo es desarrollar en los niños la habilidad para organizar temporalmente sucesos pertenecientes a este tipo de rutinas.

El contenido de la segunda unidad es la integración de la totalidad de los eventos constituyentes de los scripts cotidianos. Su objetivo es desarrollar la habilidad para organizar todas las acciones que constituyen un guión.

Las actividades sugeridas incluyen tareas de representación, ordenación y verbalización. Las de representación consisten en dramatizar rutinas completas o parte de ellas utilizando objetos y vestimentas apropiadas a la rutina. Por ejemplo, usar un delantal blanco, estetoscopio, medicamentos ficticios para simular la visita al médico. Las actividades de ordenación se refieren a disponer láminas que representan el guión en la secuencia adecuada. Así, una tarea puede ser ordenar 3 ó 4 láminas del guión acostarse. Finalmente, la verbalización requiere que los niños expresen oralmente una rutina, por ejemplo, contar la secuencia de eventos para ir de compras al supermercado.

Es necesario destacar que las actividades se diseñaron según los cambios evidenciados por cada niño en el manejo de los guiones durante el proceso de intervención.

Las sesiones terapéuticas se estructuran en tres momentos secuenciados: inicio, núcleo y final. En el inicio se establece una primera aproximación con el niño a partir de tareas que justifiquen el desarrollo del núcleo de la sesión. Luego, en el núcleo mismo se desarrollan las actividades relacionadas con los contenidos del programa terapéutico. Por último, el final consiste en realizar actividades con cuentos mediante su lectura, dramatizaciones y narración de historias. En relación a este último momento de la sesión, existe evidencia que las actividades lúdicas en torno a los cuentos inciden significativamente en el desarrollo de la competencia narrativa ${ }^{29}$.

A continuación se presenta el guión "ir al doctor" para ilustrar los tres momentos de la sesión. Este script incluye la siguiente secuencia de eventos: a) niños esperando en la sala de espera, doctor abriendo la puerta de la consulta; b) niño/niña conversando con el doctor adentro de la consulta; c) doctor examinando al niño/niña; d) doctor indica lo que hay que hacer (entrega un jarabe, cura una herida, entrega un remedio, etc. según lo que corresponde a cada niño) y e) niño/niña sale de la consulta.

Inicio. El adulto saluda a los niños sentados en círculo y propone que cada uno cuente si ha ido 
al doctor alguna vez. Puede hacer comentarios en relación a cuándo se visita al doctor, qué hace el doctor durante la visita, qué ropa usa el doctor, etc. Con esto establece la interacción inicial y da paso al desarrollo del núcleo de la sesión.

Núcleo. El adulto cuenta a los niños que van a jugar a "la visita al doctor". Entre todos acomodan la sala: un sector se transforma en sala de espera (donde esperan los niños para ser atendidos) y otro espacio funciona como consulta médica. Luego, el adulto se disfraza de doctor, saca un maletín con objetos pertinentes (jeringa, jarabe, estetoscopio, venditas, etc.) y les explica que cada uno va de visita porque está enfermo. Para ello, reparte a los niños una tarjeta con un dibujo que representa a un niño o niña que se siente mal (uno con dolor de cabeza, otro con dolor de estómago, otro con una pierna herida, otro con dolor de garganta y otro con tos). Les explica que en el dibujo está representado de qué están enfermos. Les da un ejemplo concreto con la participación de alguno de los niños, verbalizando la secuencia de acciones que forman parte del guión e inicia la actividad con todos ellos.

Al término de la actividad, los reúne y les reparte dos láminas más que ilustran la secuencia del guión completo y les pide uno a uno que verbalicen lo que hicieron.

Final. Los niños en conjunto colorean sobre un papel kraft, un cómic que representa las acciones principales del guión la visita al doctor. Después lo ponen sobre la pared de la sala.

Es importante destacar que esta intervención constituye el primer programa del plan de estimulación para el desarrollo narrativo propuesto por las autoras de este artículo ${ }^{30,31}$.

El programa se aplicó sólo al grupo experimental, durante el horario en que los niños asistían a clases en una escuela para trastornos de la comunicación oral al igual que los del grupo control. Por lo tanto, no implicó una estimulación adicional a la que ellos tienen habitualmente.

En estos establecimientos los niños reciben en forma regular una estimulación integral del lenguaje, lo que implica incrementar competencias fonológicas, morfosintácticas, semánticas y pragmáticas. Un recurso utilizado habitualmente para promover las habilidades lingüísticas señaladas es el cuento infantil. Así, los niños del grupo control pueden incrementar indirectamente su desarrollo narrativo al participar en las actividades con este tipo de textos.

La intervención se realizó individualmente en el grupo experimental y se elaboraron actividades específicas para cada niño. Se efectuaron 18 sesiones de 45 minutos cada una con una frecuencia de dos veces por semana, durante nueve semanas.
El programa se implementó en las escuelas a las que asistían los niños en una sala destinada para este fin.

El desempeño de los niños en el manejo de scripts durante la aplicación del programa se registró con el método de caso único, modelo A-B-A' ${ }^{32}$. Esto implica una primera evaluación (Fase $A$, línea de base) para establecer el rendimiento de los niños al ingresar al programa y planificar las actividades terapéuticas del inicio. Se utilizó un procedimiento específico basado en el modelo de Naremore ${ }^{18,22}$. En dicho procedimiento se usa un set de tres scripts conocidos por los niños (ducharse, comerse un yoghurt y lavarse las manos), los cuales se deben representar, ordenar y verbalizar. Las tareas utilizadas implican tres niveles: representación, ordenación y verbalización.

La representación consiste en que el niño realiza la pantomima de la secuencia de un guión. Para ello, se necesitan objetos que permitan apoyar su dramatización. Así, en "lavarse los dientes" se necesita un cepillo de dientes, un dentífrico y una toalla; en "comer un yogurt" un envase vacío con tapa, una cuchara y un basurero y en "bañarse" una toalla y un jabón. La ordenación requiere que el niño ordene láminas que representan cada acción nuclear del guión en la secuencia temporal que lo caracteriza. Para ello, se diseñaron tres láminas (cada imagen representa una acción central del guión) con un tamaño aproximado de $10 \times 15 \mathrm{~cm}$. Finalmente, en la verbalización el niño relata el guión sin el apoyo de objetos ni láminas.

La evaluación se inicia con la tarea de representación entregando la siguiente instrucción: "yo conozco un niño que no sabe lavarse los dientes y tengo que enseñarle. ¿Me puedes ayudar mostrándome qué haces cuando te los lavas?". Es importante que el niño entienda que sólo va a simular los movimientos y que no efectuará realmente el guión. Luego, se le entregan las láminas para la tarea de ordenación y se le dice: "aquí hay unos dibujos. ¿Sabes cuál es el primero?, ¿cuál sigue?, ¿cuál va al final? Podrías ponerlos en orden”. Por último, para la verbalización se le dice "ahora cuéntame lo que ordenaste". Del mismo modo se procede con los otros dos guiones.

Para corregir e interpretar los resultados de esta evaluación se asigna 1 punto a cada acción nuclear que el niño representa, ordena o verbaliza. De este modo, por cada guión es posible obtener un máximo de 9 puntos, por lo tanto, el mayor puntaje que se puede lograr es 27. A partir de este puntaje se establece la proporción de logro (respuestas correctas) de cada niño evaluado.

El rendimiento durante la aplicación del programa (Fase B) incluyó evaluaciones transterapéu- 
ticas realizadas cada cuatro sesiones. Para este fin, se utilizaron tres sets de guiones diferentes a los de la fase A con el propósito de que no influyera el recuerdo de estos en el desempeño de cada niño. El resultado de estas evaluaciones permitió el diseño de actividades para continuar con la intervención.

Por último, se estableció el rendimiento posterapéutico (Fase $A^{\prime}$ ). En este caso se utilizó el mismo set de la Fase $A$, el que se aplicó 15 días después de removida la terapia. Además, se evaluó nuevamente el desarrollo narrativo en ambos grupos de niños. Para ello, se utilizó el mismo procedimiento que en la evaluación inicial.

\section{RESULTADOS}

Una vez finalizada la aplicación del programa se comparó el desarrollo narrativo de los niños de ambos grupos. Para ello, se utilizó la prueba no paramétrica de Wilcoxon. Con el fin de ilustrar más claramente los resultados, la Tabla 1 incluye el promedio y la desviación estándar para cada grupo. Estos datos se obtienen de los puntajes asignados a los relatos de los niños, tal como se explicó en la sección participantes.

En la Tabla 1, se observa que el desarrollo narrativo del grupo experimental se incrementó significativamente al término del programa. En cambio, el grupo control no evidenció un aumento significativo en este aspecto. Es decir, el programa basado en el manejo de guiones contribuyó al desarrollo narrativo en el grupo experimental.

Según lo anterior, se estimó importante establecer si este incremento se producía en todos los niños o solo en alguno de ellos. Con este fin, se estableció la etapa de desarrollo en que se encontraban al inicio del programa y al finalizarlo. Los datos se muestran en la Figura 4.

En la Figura 4 se observa que antes de iniciar el programa, cuatro niños estaban en la etapa 1, lo que significa que no podían decir nada en la tarea de recontado. Los dos niños restantes se encontraban en la etapa 4 , es decir, expresaban oraciones en torno a un personaje.

Después de la aplicación del programa, tres niños logran estructurar sus relatos con algunas de las categorías nucleares completas (presentación

Tabla 1 - Comparación del desarrollo narrativo entre el grupo experimental y control después de la aplicación del programa para el manejo de guiones

\begin{tabular}{lccccc}
\hline & \multicolumn{2}{c}{$\begin{array}{c}\text { Rendimiento antes } \\
\text { del programa }\end{array}$} & \multicolumn{2}{c}{$\begin{array}{c}\text { Rendimiento después } \\
\text { del programa }\end{array}$} & t \\
\hline & Promedio & D.S. & Promedio & D.S. & \\
\hline $\begin{array}{l}\text { Grupo } \\
\text { Experimental }\end{array}$ & 0.63 & 1.0 & 6.13 & 4.18 & $3.72{ }^{*}$ \\
\hline Grupo Control & 0.42 & 0.26 & 2.54 & 3.66 & 1.48 n.s. \\
\hline${ }^{*} \mathrm{p}<0.05 ;$ n.s.: no significativo & & & &
\end{tabular}

\begin{tabular}{|c|c|c|}
\hline SUJETOS & ANTES DEL PROGRAMA & DESPUÉS DEL PROGRAMA \\
\hline \multicolumn{3}{|l|}{ Caso 1} \\
\hline Desarrollo narrativo & 4 & 7 \\
\hline \multicolumn{3}{|l|}{ Caso 2} \\
\hline Desarrollo narrativo & 4 & 8 \\
\hline \multicolumn{3}{|l|}{ Caso 3} \\
\hline Desarrollo narrativo & 1 & 9 \\
\hline \multicolumn{3}{|l|}{ Caso 4} \\
\hline Desarrollo narrativo & 1 & 3 \\
\hline \multicolumn{3}{|l|}{ Caso 5} \\
\hline Desarrollo narrativo & 1 & 4 \\
\hline \multicolumn{3}{|l|}{ Caso 6} \\
\hline Desarrollo narrativo & 1 & 4 \\
\hline
\end{tabular}

Figura 4 - Variación en el desarrollo narrativo en cada uno de los niños del grupo experimental 
y episodio). Por lo tanto, alcanzan un desempeño narrativo acorde a su edad.

Los otros niños, que inicialmente no decían nada, fueron capaces de expresar acciones relacionadas con el relato (dos de ellos, acciones en torno a un personaje y uno aglutinación de acciones). Aún cuando, estos participantes no logran un desarrollo de la narración apropiado para su edad, evidencian un incremento importante en relación a su rendimiento inicial. Por lo tanto, todos los niños el programa aumentan sus habilidades narrativas.

\section{DISCUSIÓN}

Los resultados obtenidos aportan antecedentes acerca de la importancia de abordar el déficit narrativo a partir de la estimulación de los guiones. Los datos previos concuerdan con otros trabajos que informan que el manejo de este tipo de rutinas es fundamental para organizar las bases del desarrollo narrativo ${ }^{18-20}$.

De este modo, estimular el manejo de guiones en niños con TEL y déficit narrativo puede ser una alternativa eficiente para mejorar las dificultades que ellos presentan en el manejo de las narraciones.

Es probable que lo anterior se relacione con las características particulares de los guiones. Estas rutinas facilitan el almacenaje y comprensión de la información, puesto que incluyen un conocimiento familiar basado en experiencias comunes para los niños ${ }^{19}$. Además, constituyen una cadena de eventos organizada de manera temporal y causal que ayudaría al niño con TEL a la comprensión de este tipo de relaciones propias de los relatos. Al respecto, se destaca la importancia de manejar el conocimiento de la causalidad para comprender que las historias implican acciones humanas que son planificadas. Por ello, es necesario que los niños manejen la organización de cadenas causales y temporales ${ }^{33}$.

Tal como se señaló en los resultados, todos los participantes del grupo experimental demostraron un mayor desarrollo narrativo al término del programa. El 50\% logró relatar con las categorías de presentación y episodio. Esto significa que la intervención les permitió alcanzar un desarrollo narrativo normal. Los demás incrementaron su desempeño, sin embargo, este no corresponde a lo esperado para su edad cronológica. Lo anterior puede deberse a que presentaban un déficit narrativo severo al inicio del programa, que se manifestaba en que no eran capaces de relatar el cuento frente a la tarea de recontado.

Con respecto al grupo control, cabe destacar que se observó un incremento en su desarrollo narra- tivo, aunque estadísticamente estas diferencias no fueron significativas al comparar su rendimiento en las dos evaluaciones realizadas. Ambas evaluaciones tuvieron la finalidad de determinar el desarrollo narrativo del grupo control en el mismo momento en que se estableció en el grupo experimental, es decir, al inicio y término de la intervención.

De este modo, la estimulación lingüística recibida por el grupo control en las escuelas de lenguaje contribuye al aumento de las habilidades narrativas, pero no es suficiente porque no les permite un desarrollo equivalente al logrado por el grupo experimental. Así, intencionar un plan de intervención específico dirigido al incremento de habilidades narrativas parece ser eficiente para reducir el déficit narrativo en los niños con TEL.

Aún cuando los resultados de esta investigación indican que la terapia de los guiones es una herramienta útil para abordar las dificultades narrativas existen dos limitaciones que es relevante destacar.

Una de ellas se refiere a la necesidad de aplicar este programa a una población más numerosa de niños con TEL, puesto que el número de participantes que recibió la intervención en este trabajo fue reducido.

La otra limitación es que no se consideró un período de seguimiento de los participantes una vez finalizada la estimulación lo que permitiría verificar el efecto de la terapia en el tiempo. Por lo tanto, es recomendable realizar investigaciones prospectivas que incluyan un período de evaluación posterior al término de la terapia para constatar si los niños mantienen el ritmo de desarrollo narrativo que evidenciaron durante la intervención.

Según las limitaciones planteadas, existen aspectos que es necesario considerar en futuras investigaciones. Sin embargo, los hallazgos de este trabajo sugieren que es posible favorecer tempranamente el discurso narrativo en preescolares con TEL con un plan específico de intervención. La superación de las dificultades narrativas en la etapa preescolar contribuye, en el período escolar, a que ellos enfrenten el aprendizaje del lenguaje escrito con mayores posibilidades de éxito. La razón de ello se sustenta en que la calidad de las narraciones de los escolares refleja el uso de un lenguaje cada vez más complejo y el nivel de alfabetización que han alcanzado ${ }^{34}$.

\section{CONCLUSIÓN}

Los resultados de este trabajo permiten concluir que la implementación de un programa de estimulación de las habilidades narrativas iniciales resulta eficaz para los niños con trastorno específico de lenguaje y déficit narrativo. El planteamiento previo 
se basa en que la mayoría de los niños que recibieron el programa evidenciaron un mayor desarrollo narrativo al compararlos con los del grupo control.

Esta evidencia es útil en la planificación de programas de intervención en niños con problemas de lenguaje pues indica la posibilidad de incorporar el discurso narrativo como un contenido específico desde la etapa preescolar, contribuyendo de este modo a que los niños tengan mayores competencias para enfrentar el aprendizaje escolar.

\begin{abstract}
Purpose: providing evidence about the possible contribution of scripts to the narrative development of a group of children with Specific Language Impairment and narrative deficit. Methods: a program for script-based narrative development intervention is submitted. Twelve preschool children with Specific Language Impairment participated in the current study. They were divided at random in two groups. The intervention was developed in one of these groups and, the other was the control group (mean age of 4;5 for both groups). Results: all children in the experimental group significantly increased their narrative development. Including $50 \%$ of them that achieved a narrative level corresponding to their chronological age. No significant increase was noted in the control group. Conclusion: the present study shows that using scripts in children with Specific Language Impairment and narrative deficit may be successful in helping them to overcome their difficulties in narrative performance.
\end{abstract}

KEYWORDS: Language Disorders; Narration; Language Therapy

\section{REFERÊNCIAS}

1. Fresneda MD, Mendoza E. Trastorno específico del lenguaje: concepto, clasificaciones y criterios de identificación. Rev Neurol. 2005; 41(Supl1):S51-S6.

2. Tomblin JB, Records NL, Bukwalter P, Zhang X, Smith E, O'Brien M. Prevalence of specific language impairment in kindergarten children. J Speech Lang Hear Res. 1997; 40(6):1245-60.

3. Muñoz-Yunta JA, Palau-Baduell M, SalvadóSalvadó B, Rosendo-Moreno N, Valls-Santasusana A, Perich-Alsina $X$, et al. Trastornos específicos del lenguaje: diagnóstico, tipificación y estudios con magnetoencefalografía. Rev Neurol. 2005; 40(Supl1):S115-S9.

4. Crespo-Aguilaz N, Narbona J. Perfiles clínicos evolutivos y transiciones en el espectro del trastorno específico del desarrollo del lenguaje. Rev Neurol. 2003; 36(Supl1):S29-S35.

5. Muñoz-López J, Carballo-García G. Alteraciones lingüísticas en el trastorno específico del lenguaje. Rev Neurol. 2005; 41(Supl1):S57-S63.

6. Im-Bolter $\mathrm{N}$, Johnson $\mathrm{J}$, Pascual-Leone J. Processing limitations in children with specific language impairment: the role of executive function. Child Develop. 2006; 77(6):1822-41.

7. Archibald LMD, Gathercole SE. Visuospatial immediate memory in specific language impairment. J Speech Lang Hear Res. 2006; 49(2):265-77.
8. Engel S. The narrative worlds of "what is" and "what if". Cognit Develop. 2005; 20(4):514-25.

9. Paul R, Smith RL. Narrative skills in 4-yearsolds with normal, impaired, and late-developing language. J Speech Hear Res. 1993; 36(3):592-8.

10. Bishop DVM, Edmundson A. Language-impaired 4-year-olds: distinguishing transient from persistent impairment. J Speech Hear Disord. 1987; 52(2): 156-73.

11. Liles BZ. Narrative discourse in children with language disorders and children with normal language: a critical review of the literature. J Speech Hear Res. 1993; 36(5):868-82.

12. Liles BZ, Duffy RJ, Merrit DD, Purcell SL. Measurement of narrative discourse ability in children with language disorders. J Speech Hear Res. 1995; 38(2):415-25.

13. Kaderavek JN, Sulzby E. Narrative production by children with and without specific language impairment: oral narratives and emergent readings. J Speech Lang Hear Res. 2000; 43(1):34-49.

14. Swanson LA, Fey ME, Mills CE, Hood LS. Use of narrative-based language intervention with children who have specific language impairment. Am J Speech Lang Path. 2005; 14(2):131-43.

15. Pavez MM, Coloma CJ, González P, Palma $\mathrm{SM}$, Reinoso CN. El discurso narrativo en niños con trastorno específico del lenguaje. Rev Chil Fonoaudiol. 1999; 1(2):17-32.

16. Pavez MM, Coloma CJ. Desarrollo del discurso en niños con trastorno específico del lenguaje. 
En: Harvey A, organizador. En torno al discurso. Santiago de Chile: Ediciones Universidad Católica de Chile; 2005. p. 149-56.

17. Mistry JJ, Lange GW. Children's organization and recall of information in scripted narratives. Child Develop. 1985; 56(4):953-61.

18. Naremore RC. Making it hang together: children's use of mental frameworks to structure narratives. Topics Lang Disord. 1997; 18(1):16-30.

19. Owens R. Language disorders: a functional approach to assessment and intervention. 4. ed. Boston: Pearson Education Inc; 2004. 571p.

20. Hughes D, McGillivray L, Schmideck M. Guide to narrative. Procedures for assessment. Wisconsin: Thinking Publication; 1997. 327p.

21. Paul R. Language disorders from infancy through adolescence: assessment \& intervention. 2. ed. Saint Louis: Mosby; 2001. 650p.

22. Naremore R, Densmore A, Harman D. Assessment and treatment of school age language disorders: a resource manual. Canada: Singular Thomson Learning; 2001. 191p.

23. Burgemeister BB, Blum LH, Lorge I. Escala de madurez mental de Columbia. Madrid: TEA Ediciones; 2004. 53p.

24. Maggiolo M, Pavez MM. Test para evaluar procesos fonológicos de simplificación. Santiago de Chile: Ediciones Escuela de Fonoaudiología; 2000. 62p.

25. Pavez MM. Test exploratorio de gramática española de A. Toronto. Santiago de Chile: Ediciones Universidad Católica de Chile; 2003. 46p.

26. Carrasco L, Urrutia C, Zelada G. Aplicación del Test de Carrow a una muestra de niños chilenos de 3 a 4 años 11 meses. Seminario de investigación para optar al Título de Fonoaudiólogo, tutoreado por María Mercedes Pavez, Escuela de Fonoaudiología, Universidad de Chile; 1983.

27. Hess L. I would like to play but I don't know how: a case study of pretend play in autism. Child Lang Teach Ther. 2006; 22(1):97-116.

28. Maggiolo M, Pavez MM, Coloma CJ. Terapia para el desarrollo narrativo en niños con trastorno específico del lenguaje. Rev Logop Foniatr Audiol. 2003; 23(2):98-108.

29. Baumer S, Ferholt B, Lecusay R. Promoting narrative competence through adult-child joint pretense: lessons from the Scandinavian educational practice of playworld. Cognit Develop. 2005; 20 : 576-90.

30. Ilgaz H, Aksu-Koc A. Episodic development in preschool children's play-prompted and directelicited narratives. Cognit Develop. 2005; 20(4): 526-44.

31. Pavez MM, Coloma CJ, Maggiolo MM. El desarrollo narrativo en niños. Evaluación y estimulación. Barcelona: Ars Médica; 2008. 221 p.

32. Barlow D, Heusen M. Diseños experimentales de caso único. Barcelona: Ed. Martínez Roca; 1998. $159 \mathrm{p}$

33. Klecan-Aker JS, Flahive L, Fleming S. Teaching storytelling to a group of children with learning disabilities: a look at treatment outcomes. Contemp Issues Commun Sci Dis. 1997; 24:23-32.

34. Ukrainetz TA, Justice LM, Kaderavek JN, Eisenberg SL, Gilliam RB, Harm HM. The development of expressive elaboration in fictional narratives. J Speech Lang Hear Res. 2005; 48(6):1363-77.

DOI: $10.1590 / S 1516-18462009005000043$

RECEBIDO EM: 19/08/2008

ACEITO EM: 14/06/2009

Endereço para correspondência:

Mariângela Maggiolo Landaeta

Rua Independencia, 1027 casilla 13898

Santiago, Región Metropolitana

Fax: (56) (2) 978-6608

E-mail: mmaggiol@med.uchile.cl 